\title{
Dissecação e Capacitação de Habilidades e Competências Gerais na Formação Médica
} Dissection and Training of Skills and General Competences in Medical Education

\author{
AMIRA ROSE COSTAMEDEIROS ${ }^{1}$ \\ EULÂMPIO JOSÉ DA SILVANETO² \\ MATHEUS GURGEL SARAIVA ${ }^{3}$ \\ LUIZ ALBERTO SOARES DE ARAÚJO COUTINHO ${ }^{3}$ \\ ANTONIO COUTINHO MADRUGA NETO ${ }^{3}$ \\ ROSA-MARIA SILVA SOARES ${ }^{3}$ \\ CAMILAMARQUES PEREIRA ALENCAR ${ }^{4}$ \\ ISABEL GOMES NOGUEIRA VIEIRA ${ }^{4}$ \\ ÉRIKA CARDOSO SILVA4
}

\section{RESUMO}

Objetivo: Relacionar o ensino e a aprendizagem da disciplina de Dissecação na Anatomia Topográfica com a capacitação das habilidades gerais instituídas pelas Diretrizes Curriculares Nacionais dos Cursos de Medicina: atenção à saúde, tomada de decisões, comunicação, liderança, administração e gerenciamento, e educação permanente. Materiais e Métodos: Trata-se de um estudo seccional que envolveu uma população de 270 alunos do curso de Medicina da Faculdade de Medicina Nova Esperança e da Universidade Federal da Paraíba, ambas em João Pessoa - PB. A amostra foi selecionada aleatoriamente e incluiu 132 graduandos, que responderam a um questionário estruturado desenvolvido pelos autores. Resultados: Não houve diferença significativa quanto ao sexo dos alunos entrevistados, tendo sido a média de idade de 21,7 anos. Dos 132 graduandos, houve o relato de que, frequente ou muito frequentemente, ocorreu o desenvolvimento de: capacidade de tomar decisões $-57,2 \%$, liderança - 57,7\%; administração e gerenciamento - $74,3 \%$, comunicação - $87,1 \%$, educação continuada - $76,0 \%$, atenção à saúde - 59,8\%. Conclusão: A dissecação tem importância na capacitação das habilidades gerais no ensino de Anatomia Topográfica e, portanto, pode contribuir com a formação do perfil do profissional médico preconizado pelas Diretrizes Curriculares Nacionais dos Cursos de Medicina.

\section{DESCRITORES}

Anatomia. Dissecação. Educação.

\begin{abstract}
Objective: To associate teaching and learning in the subject Dissection within the field of topographical anatomy with the training of general skills established by the National Curriculum Guidelines for Medical Courses: health care, decision making, communication, leadership, administration and management, and continuing education. Material and Methods: This was a cross-sectional study involving a population of 270 students of Medicine, Nova Esperança Faculty of Medicine and the Federal University of Paraíba, both in the city of Joao Pessoa - PB. The sample was composed of 132 undergraduates randomly selected, who answered a structured questionnaire developed by the authors. Results: There was no significant difference in the gender of the students interviewed, which had mean age of 21.7 years. Of the 132 undergraduate students, often or very often, there was the development of: decision-making abilities $-57.2 \%$, leadership qualities - $57.7 \%$; administration and management $-74.3 \%$, communication $-87.1 \%$, continuing education $-76.0 \%$, health care attention $-59.8 \%$. Conclusion: Anatomic dissection is important in the training of general skills in the teaching of Topographical Anatomy and hence can contribute to the formation of the medical profile recommended by the National Curriculum Guidelines for Medical Courses.
\end{abstract}

DESCRIPTORS

Anatomy. Dissection. Education.

1 Médica cardiologista. Mestrado em Enfermagem de Saúde Pública. Professora Assistente de Anatomia na UFPB. Professora da Faculdade de Medicina Nova Esperança, João Pessoa/PB, Brasil.

2 Médico veterinário. Doutor em Anatomia Comparada de Vertebrados. Professor Associado de Anatomia da Universidade Federal da Paraíba (UFPB), João Pessoa/PB, Brasil.

3 Graduando (a) de medicina da Universidade Federal da Paraíba (UFPB), João Pessoa/PB, Brasil.

4 Graduanda de medicina da Faculdade de Medicina Nova Esperança, João Pessoa/PB, Brasil. 
$\mathrm{E}$ 2001, O Conselho Nacional de Educação, através da Câmara de Educação Superior, instituiu Resolução CNE/CES n ${ }^{\circ}$ 4, de 07 de novembro de 2001, que trata das Diretrizes Curriculares Nacionais do Curso de Medicina (CNE/CES, 2001).

Esta Resolução institui em seu Art. $4^{\circ}$ que "a formação do médico tem por objetivo dotar o profissional dos conhecimentos requeridos para o exercício das seguintes competências e habilidades gerais:

I- Atenção à saúde: "devem estar aptos a desenvolver ações de prevenção, promoção, proteção e reabilitação da saúde"

II- Tomada de decisões: "capacidade de tomar decisões visando eficácia e custo-efetividade (...) de procedimentos e práticas. Possuir competências e habilidades para avaliar, sistematizar e decidir as condutas mais adequadas"

III- Comunicação: "profissionais de saúde devem ser acessíveis (...), envolve comunicação verbal, não verbal e habilidades de escrita e leitura"

IV - Liderança: "assumir posições de liderança, (...) envolve compromisso, responsabilidade, empatia, habilidade para decisões, comunicação e gerenciamento"

V - Administração e gerenciamento: "devem estar aptos a tomar iniciativas, fazer gerenciamento dos recursos físicos e materiais e de informação"

VI- Educação permanente: "capazes de aprender continuamente, (...) aprender a aprender e ter responsabilidade e compromisso com sua educação" (CNE/CES, 2001).

Neste contexto, o ensino da Anatomia Humana através da técnica dissecatória em grupos de alunos parece ser uma ferramenta importante no desenvolvimento das habilidades e competências descritas. Sabe-se que o estudo da anatomia topográfica pode ser melhor realizado quando o próprio aluno tem a oportunidade de dissecar a região a ser estudada, pois ele é capaz de identificar os planos anatômicos e as estruturas da região, ao tempo em que aperfeiçoa a técnica dissecatória, muito importante para os procedimentos médicos (WEBER, 2001).

Através da dissecação a construção do saber ocorre como processo ativo, no qual o aluno interage e atua na produção do conhecimento. Dependendo de como a dissecação é realizada, pode ser considerada uma ferramenta da abordagem cognitivista de ensino, já que a aquisição de conhecimento deve-se ao exercício e experiência física, implicando-se, segundo MIZUKAMI (1986), em "agir sobre objetos para descobrir as propriedades que são abstraídas destes próprios objetos".
O processo de ensino-aprendizagem da anatomia topográfica através da dissecação além de propiciar ao aluno a obtenção do conhecimento anatômico propriamente dito, permite a atividade em grupo, a melhor interação entre professor-aluno, a troca de experiências entre os grupos, o desenvolvimento de habilidades de decisão, liderança, aprendizado auto-didata e comunicação entre os profissionais.

Haja vista a importância da dissecação no processo de ensino aprendizagem do estudante da área de saúde, sobretudo nos cursos médicos, ainda não é uma prática amplamente utilizada nas faculdades de medicina, principalmente pela dificuldade em conseguir cadáveres humanos para dissecação e estudo.

O objetivo do trabalho foi avaliar a opinião de alunos que praticam a dissecação durante o estudo da anatomia topográfica, correlacionando as atividades de dissecação desenvolvidas na disciplina com a capacitação das habilidades e competências gerais instituídas nas Diretrizes Curriculares Nacionais do Curso de Medicina, e com habilidades específicas da disciplina.

\section{MATERIAIS E MÉTODOS}

Realizou-se estudo seccional e observacional, com abordagem quantitativa, através da pesquisa de campo utilizando-se a aplicação de questionário (ROUQUAYROL, 2013).

A população foi composta por alunos do curso de medicina da Faculdade de Medicina Nova Esperança (FAMENE) e da Universidade Federal da Paraíba (UFPB), ambas em João Pessoa - PB, sendo na primeira, todos os alunos do $2^{\circ}$ ao $4^{\circ}$ período e na UFPB estudantes do $4^{\mathrm{o}}$ ao $6^{\circ}$ períodos, totalizando 120 e 150 acadêmicos, respectivamente. Em ambas as instituições os alunos praticam rotineiramente a dissecação durante o estudo de anatomia topográfica. A amostra aleatória simples foi calculada considerando erro amostral de 6\%, nível de confiança de $95 \%$ e estimando-se percentual de situações que desenvolvem habilidades gerais superior a $60 \%$ (SANTOS, 2013). Os alunos foram selecionados entre aqueles que cursavam o $2^{\circ}, 3^{\circ}$ ou $4^{\circ}$ períodos do referido curso na FAMENE e do $4^{\circ}, 5^{\circ}$ e $6^{\circ}$ períodos de medicina da UFPB, sendo a diferença de períodos entre os grupos justificada pelo fato da dissecação ser oferecida na FAMENE desde o primeiro período, enquanto que na UFPB o início é a partir do terceiro período do curso. Foram incluídos na pesquisa estudantes que aceitaram participar da pesquisa assinando o Termo de Consentimento Livre e Esclarecido, respeitando as observâncias éticas 
contempladas na Resolução n 196/96 (BRASIL, 1996).

A pesquisa foi realizada inicialmente na FAMENE, onde a coleta de dados ocorreu em abril de 2008. Posteriormente os pesquisadores conduziram a mesma pesquisa na UFPB, onde se realizou a coleta dos dados em maio de 2011, ocorrendo esta diferença de datas em decorrência dos trâmites para formação da equipe e aprovação do projeto na UFPB. Em ambas as instituições a coleta foi realizada por estudantes de medicina das respectivas universidades. Os resultados foram transcritos para planilha de armazenamento de dados do Programa SPSS 17.0 for Windows Standard Version e a análise estatística constou de descrição da frequência das variáveis.

\section{RESULTADOS}

Foram incluídos um total de 132 acadêmicos de medicina, sem predomínio significativo entre os sexos (feminino - 49,2\% e masculino - 50,8\%), com idade que variou de 18 a 29 anos, com média de 21,7 anos. Quando questionados se gostavam da disciplina de anatomia, $82,6 \%$ responderam afirmativamente. Quarenta acadêmicos $(30,3 \%)$ haviam cursado outras faculdades na área de saúde, dos quais 6 indivíduos já haviam praticado dissecação em outras instituições.

Quando questionados sobre a capacitação das habilidades e competências gerais instituídas nas Diretrizes Curriculares Nacionais do Curso de Medicina, durante a disciplina Anatomia Topográfica, observouse que em todas as variáveis estudas mais da metade dos alunos afirmaram que elas foram desenvolvidas pelo estudo da dissecção, entre elas destacando-se a Comunicação, onde $87,1 \%$ dos estudantes declaram que a disciplina "frequente" ou "muito frequentemente" desenvolveu esta habilidade. (Tabela 1).

Quando questionados sobre a capacitação de habilidades e competências específicas da disciplina Anatomia Topográfica, encontrou-se que o acréscimo ao aprendizado global da disciplina foi descrito como "muito" por mais de 2/3 da amostra, quando comparada com o estudo apenas em peças previamente preparadas. Mais de $50 \%$ dos alunos que fizeram parte do estudo referiram que a dissecação desenvolveu "muito" sua habilidade técnica para manuseio de instrumental cirúrgico e realização de procedimentos cirúrgicos como fazer incisões, rebater pele e fáscias, isolar estruturas, definir trajetos de vasos e nervos (Tabela 2).

\begin{tabular}{|c|c|c|c|c|c|c|}
\hline \multirow[b]{2}{*}{ Habilidade Geral } & \multicolumn{6}{|c|}{ Frequéncia desenvolvida na dissecaçăo (\%) } \\
\hline & Nunca & Raramente & $\begin{array}{c}\text { Pouco } \\
\text { frequente }\end{array}$ & Frequente & $\begin{array}{c}\text { Muito } \\
\text { frequente }\end{array}$ & Total \\
\hline Tomada de decisões & 0.8 & 6.9 & 35.1 & 51.9 & 5.3 & 100 \\
\hline Capacidade de Ideranca & 0.0 & 9.2 & 33.1 & 48.5 & 9.2 & 100 \\
\hline $\begin{array}{l}\text { Administraçăo e } \\
\text { gerenciamento }\end{array}$ & 0,8 & 3,8 & 21,2 & 57,6 & 16,6 & 100 \\
\hline Comunicacão & 1,5 & 0.8 & 10.6 & 46.2 & 40,9 & 100 \\
\hline Educacão Dermanente & 1.6 & 3.8 & 18.6 & 54.3 & 21.7 & 100 \\
\hline Atencăo à saúde & 1,6 & 13,6 & 25.0 & 40,9 & 18,9 & 100 \\
\hline
\end{tabular}

Tabela 2. Distribuiçăo da opiniấo de graduandos de medicina quanto à frequéncia de situaçoes que desenvolvem as Habilidades Especificas de Anatomia Topográfica durante a dissecacăo.

\begin{tabular}{lcccc}
\hline $\begin{array}{l}\text { Habilidades especificas da disciplina } \\
\text { anatomia topoarafica }\end{array}$ & \multicolumn{4}{c}{$\begin{array}{c}\text { \% dos alunos frequéncia } \\
\text { desenvolvida na dissecacão }(\%)\end{array}$} \\
\cline { 2 - 5 } & Pouco & Recular & Muito & Total \\
\hline $\begin{array}{l}\text { Desernolvimento da capacidade da } \\
\text { técnica de dissecacáo }\end{array}$ & 5,3 & 43,2 & 51,5 & 100 \\
$\begin{array}{l}\text { Acréscimo ao aprendizado global da } \\
\text { Anatomia Topocráfica }\end{array}$ & 5,3 & 26,5 & 68,2 & 100 \\
\hline
\end{tabular}


Quando questionados a respeito da operacionalização da disciplina de anatomia topográfica, demonstrou-se que: 50 alunos $(37,9 \%)$ julgaram inadequado o número de professores durante as práticas de dissecação, destes 38 alunos eram da UFPB e 12 alunos da FAMENE, e 21 alunos $(15,9 \%)$ consideraram que o tempo destinado para a prática dissecatória foi insuficiente, destes 19 alunos eram da UFPB e 2 alunos da FAMENE.

\section{DISCUSSÃO}

Os resultados demonstram que há uma opinião favorável dos estudantes, que compõem a amostra, quanto à importância da dissecação na formação das habilidades gerais do médico. Percebe-se que o processo de dissecação é capaz de propiciar uma situação de atividade em grupo e de auto-aprendizado muito relevante nesta formação.

A presença de situações capazes de treinar as habilidades gerais durante a dissecação foi considerada "frequente ou muito frequente" por mais de $50 \%$ dos alunos para todas elas, com descrição superior a $70 \%$ dos alunos para capacidade de comunicação, de educação continuada e de administração e gerenciamento, que são habilidades muito presentes durante a prática dissecatória.

Este estudo corrobora os resultados descritos por RAMSEY-STEWART (2010), que avaliou a opinião de 29 estudantes de medicina que praticaram dissecação em uma Universidade de Sydney. Neste estudo todos os estudantes classificaram o curso de dissecação como "muito bom", e foi recomendado unanimemente que o curso fosse disponibilizado para todos os estudantes como parte do currículo médico.

Em uma completa e atual revisão sobre a importância da dissecação versus os aspectos práticos para sua implantação rotineira nos cursos médicos, FAZAN (2011) destaca a importância da observação, identificação e interpretação propiciadas pela dissecação como base na formação médica, e ressalta que em países desenvolvidos como os EUA, 97\% das faculdades de medicina utilizam-se da dissecação como ferramenta no ensino de anatomia. A disponibilidade de cadáveres e a falta de incentivo à doação continuam sendo os fatores limitantes principais (DAVIM, 2010).

De acordo com WEBER (2011), o estudo da anatomia topográfica pode ser mais bem realizado quando o próprio aluno tem a oportunidade de dissecar a região a ser estudada, pois ele é capaz de identificar os planos anatômicos e as estruturas da região, ao tempo em que aperfeiçoa a técnica dissecatória, muito importante para os procedimentos médicos.

Numa pesquisa realizada por POCHAT (2011), com residentes de Cirurgia Plástica do Hospital Universitário Professor Edgard Santos (HUPES) da Universidade Federal da Bahia em convênio com o Instituto Médico Legal do Estado da Bahia, de 2009 a 2010, avaliou-se a importância da dissecação de cadáveres no treinamento dos residentes do serviço, diminuindo a curva de aprendizado em cirurgias de maior complexidade, elevando a qualidade do atendimento prestado e promovendo melhor assistência à população.

Neste estudo, apesar dos resultados favoráveis em relação ao desenvolvimento das habilidades gerais na formação dos estudantes, foram demonstrados fatores limitantes ao ensino da dissecação. Do total, 21 $(15,9 \%)$ alunos consideraram insuficiente o tempo destinado para a prática dissecatória, destes 90,5\% (19 alunos) eram da UFPB. Essa queixa com relação ao tempo, foi inferior ao encontrado numa revisão de literatura realizada por FORNAZIERO (2003), no qual uma pesquisa com 727 estudantes demonstrou que $26 \%$ destes referiram a necessidade de incrementar as aulas práticas com maior quantidade de horas dedicadas aos trabalhos práticos com cadáveres e uso de melhor material didático.

Este resultado pode sugerir reduzida carga horária dos professores destinada À dissecação por sobrecarga nas atividades de ensino tradicional, bem como limitações operacionais quanto à obtenção de cadáveres e material de técnica dissecatória, além da falta de estímulo e incentivo. 
Segundo TAVANO (2011), "um número maior de cursos, instituições e estudantes em curto espaço de tempo dificulta a obtenção de cadáveres humanos em quantidade e qualidade suficientes para o estudo". Além disso, o uso constante com muitos manipuladores pode levar à perda acentuada do material em intervalos menores de tempo.

Haja vista a importância da dissecação no processo de ensino e aprendizagem do estudante da área de saúde, sobretudo nos cursos médicos, esta ainda não é uma prática amplamente utilizada nas faculdades de medicina, principalmente pela dificuldade em conseguir cadáveres humanos para dissecação e falta de incentivo à doação. Neste estudo, outros fatores limitantes observados foram o tempo insuficiente destinado às práticas de dissecação, assim como a indisponibilidade de professores da disciplina de Anatomia.

\section{CONCLUSÃO}

A dissecação tem importância fundamental no aprendizado da Anatomia Topográfica e na capacitação das habilidades e competências gerais para a formação do médico, instituídas pela Resolução CNE/CES n ${ }^{\circ}$ / 2001, segundo a opinião de acadêmicos do curso de medicina da Faculdade de Medicina Nova Esperança e da Universidade Federal da Paraíba.

Através da dissecação é possível desenvolver as habilidades específicas da disciplina anatomia topográfica, criar habilidade no manuseio de instrumental cirúrgico, e aliar-se à capacitação de habilidades de cunho psico-social, que tornam o aprendizado mais efetivo, mais ativo por parte do aluno e aproximam o binômio professor-aluno, permitindo maior troca de experiências e melhor construção do conhecimento anatômico.

Estes resultados têm especial relevância no contexto de mudança de grade curricular dos cursos de medicina em virtude das novas diretrizes e demonstram que a dissecação pode contribuir com a formação do perfil do profissional médico almejado pelas Diretrizes Curriculares Nacionais do Curso de Medicina.

\section{REFERÊNCIAS}

1. BRASIL. Ministério da Saúde. Conselho Nacional de Saúde. Resolução № 196/96, 10 de outubro de 1996 dispõe sobre pesquisa envolvendo seres humanos. Brasília, DF, 1996.

2. CNE/CES - CONSELHO NACIONAL DE EDUCAÇÃO. Câmara de Educação Superior. Resolução CNE/CES 4/ 2001. Diretrizes Curriculares Nacionais do Curso de Medicina. Diário Oficial da União, Brasília, 9 de novembro de 2001. Seção 1, p.38.

3. DAVIM LS. Doação de Cadáveres para Estudos Acadêmicos em Discussão. O Anatomista - Rev de Divulgação Científica da Soc Bras de Anat. 2010; 1(2): 4-5.

4. FAZAN VPS. Métodos de ensino em anatomia: dissecção versus prossecção. O Anatomista - Rev de Divulgação Científica da Soc Bras de Anat. 2011; 2(1): 7-12.

5. FORNAZIERO CC, GIL CRR. Novas Tecnologias Aplicadas no Ensino da Anatomia Humana. Rev Bras Educ Med, 27(2): 141-6 , 2003.

6. MIZUKAMI MGN. Ensino: as abordagens do processo, São Paulo: EPU, 66-71, 1986.

7. POCHAT VD, MENDES RRS, FIGUERÊDO AA, CUNHA NAMS, MENESES JVL. Atividades de dissecção de cadáveres e residência médica: relato da experiência do Serviço de Cirurgia Plástica do Hospital Universitário Professor Edgard Santos da Universidade Federal da Bahia. Rev. Bras. Cir. Plást. 2011; 26(4): 561-5.

8. RAMSEY-STEWART G, BURGESS AW, HILL DAL. Back to the future: teaching anatomy by whole-body dissection. Med J Aust. 2010; 193 (11): 668-671.

9. ROUQUAYROL MZ, GURGEL M. Epidemiologia \& Saúde. $7^{\text {a }}$ Ed, Rio de Janeiro: MedBook, 2013. 
10. SANTOS, Glauber Eduardo de Oliveira. Cálculo amostral: calculadora on-line. Disponível em: <http:// www.calculoamostral.vai.la>. Acesso em: [19/04/2011].

11. TAVANO PT, ALMEIDA MI. A reconfiguração do Ensino Anatômico: Tensões que Incidem na Disciplina Básica. Rev Bras Educ Med. 2011; 35(3): 421-428.

12. WEBER JC. Manual de dissecação humana de Shearer, 8ª ed, São Paulo: Editora Manole Ltda, 2001.
Correspondência

Amira Rose Costa Medeiros

Av. Hilton Souto Maior, 6701, Quadra 755, Lote 239, Portal do Sol

João Pessoa - Paraíba - Brasil

CEP 58046-900

E-mail: amiramedeiros@gmail.com 